\title{
BMJ Open Advance care planning for vulnerable older adults within an Accountable Care Organization: study protocol for the IMPACT randomised controlled trial
}

\author{
Jennifer Gabbard (D , ${ }^{1}$ NM Pajewski, ${ }^{2}$ Kathryn E Callahan, ${ }^{1}$ Ajay Dharod, ${ }^{3}$ \\ Kristie Foley, ${ }^{4}$ Keren Ferris, ${ }^{5}$ Adam Moses, ${ }^{3}$ Carl Grey, ${ }^{1}$ Jeff Williamson ${ }^{1}$
}

To cite: Gabbard J, Pajewski NM, Callahan KE, et al. Advance care planning for vulnerable older adults within an Accountable Care Organization: study protocol for the IMPACT randomised controlled trial. BMJ Open 2019;9:e032732. doi:10.1136/ bmjopen-2019-032732

- Prepublication history and additional material for this paper are available online. To view these files, please visit the journal online (http://dx.doi. org/10.1136/bmjopen-2019032732).

Received 03 July 2019 Revised 13 November 2019 Accepted 15 November 2019

Check for updates

(C) Author(s) (or their employer(s)) 2019. Re-use permitted under CC BY-NC. No commercial re-use. See rights and permissions. Published by BMJ.

For numbered affiliations see end of article.

Correspondence to Dr Jennifer Gabbard; jgabbard@wakehealth.edu

\section{ABSTRACT}

Introduction Patients with multimorbidity plus additional impairments (eg, mobility limitations, disability, cognitive impairments or frailty) are at the highest risk for poor healthcare outcomes. Advanced care planning (ACP) provides patients and their surrogates the opportunity to discuss their goals, values and priorities for healthcareparticularly in the context of end-of-life care. ACP discussions promote more person-centred care; however, it is currently underused. There is a tremendous need for systematic, scalable approaches to individualised ACP that promotes patient and family engagement. Here we describe the study protocol for a randomised effectiveness trial of a nurse navigator and informatics intervention designed to improve the documentation and quality of ACP discussions.

Methods and analysis This is a randomised, pragmatic, effectiveness trial; patients aged 65 years and older who have multimorbidity plus impairments in either physical function (eg, mobility limitations or disability) or cognition, and/or frailty within an affiliated Accountable Care Organization were eligible. The electronic health record was used to develop an automatic prescreening system for eligible patients $(n=765)$ and participants were randomised in a 1:1 ratio to either the nurse navigator-led ACP pathway or usual care. Our primary outcomes are documentation of ACP discussions within the EHR along with the quality of ACP discussions. Secondary outcomes include a broad range of ACP actions (eg, usage of ACP billing codes, choosing a surrogate decision-maker and advance directive documentation). Outcomes will be measured over 12 months of follow-up.

Ethics and dissemination This study has been approved by the appropriate Institutional Review Boards and is guided by input from patient and clinical advisory boards. The results of this study will inform a scalable solution to ACP discussions throughout our healthcare system and statewide.

Trials registration number NCT03609658.

\section{INTRODUCTION}

One-fifth of the total US population will be over the age of 65 by $2050 .^{12}$ Inevitably, there will be a corresponding surge in those with multiple chronic conditions
Strengths and limitations of this study

This study addresses gaps in advance care planning (ACP) for at-risk, vulnerable older adults.

- An automatic prescreening system was designed to identify vulnerable older adults within the electronic health record (EHR) to improve recruitment.

- Nurse navigators are used in this study for ACP previsit planning over the telephone to improve patient engagement.

- Integrating provider facing EHR ACP tools is an innovative method to improve ACP discussions, documentation and promote engagement.

- This study is only occurring within an Accountable Care Organization population in North Carolina, thus may have limited generalisability.

('multimorbidity') along with an associated increase in healthcare expenditures. ${ }^{1-6}$ Multimorbidity has been associated with (1) poor patient health outcomes, including depression, polypharmacy, socioeconomic deprivation, poorer quality of life and decreased satisfaction with care; and (2) increased overall health system costs, primarily due to increased healthcare utilisation and burdensome care. ${ }^{7-18}$ Yet, multimorbidity alone does not identify the subset of older adults at greatest need of assistance with care planning. ${ }^{19}{ }^{20}$ Evidence is emerging that persons with multimorbidity plus impairments in either function, cognition and/or frailty are at the highest risk for poor outcomes with respect to disability and mortality, above and beyond the risk attributable to individual diseases. $^{2}{ }^{18} 20-25$ Here, we label these patients as vulnerable older adults: adults 65 years and older who have multimorbidity plus impairments in either physical function (eg, mobility disability), cognition and/or frailty. At present, the care of vulnerable older adults is marked by fragmented healthcare 


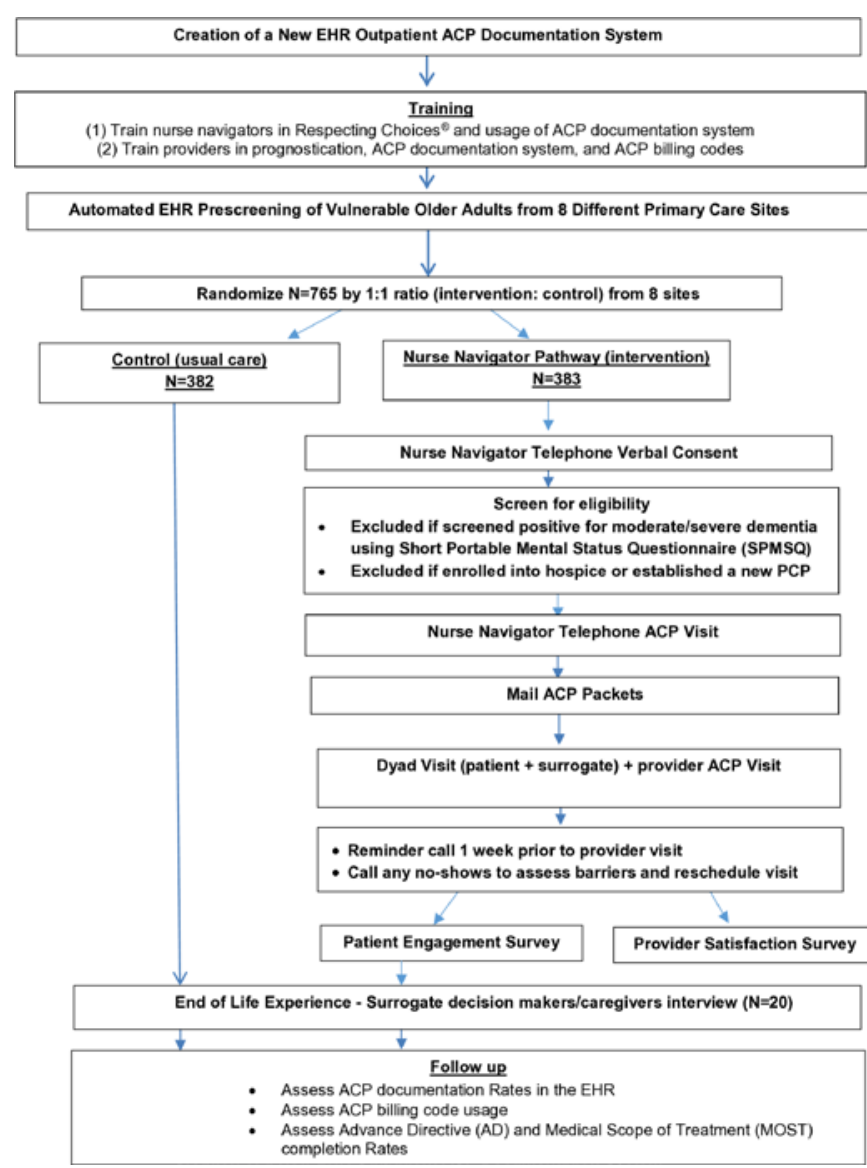

Figure 1 IMPACT study flow diagram. IMPACT, Integrated Multidisciplinary Patient and Family Advance Care Planning Trial, ACP, advance care planning; EHR, electronic health record; PCP, primary care provider.

focused on disease-based treatments, lengthy and recurrent hospital stays and higher healthcare cost through the end of life. ${ }^{26-29}$ Studies have shown that older adult's preserved functional health status is a prerequisite for higher quality of life, and functional decline is a strong prognostic indicator. ${ }^{25} 3031$ As opposed to a disease-based approach to healthcare, a function-based and goal-based approach for patients at risk for worse outcomes can help inform advance care planning in vulnerable older adults. $^{32}$

The use of patient-level variables that are gathered during routine medical care within the electronic health record (EHR) allows for easier patient identification for implementing pragmatic clinical trials. ${ }^{33}$ Recruiting patients directly from the EHR allows for prescreen eligibility prior to approaching potential participants to help facilitate patient recruitment. ${ }^{34}$ Thus, we propose to promote advance care planning (ACP) conversations by first using the EHR for automatic prescreening for vulnerable older adults, and then developing a new outpatient ACP documentation system that promotes easy documentation along with providing a central location for documented goals of care discussions within the EHR. Second, we will leverage nurse navigators as the first point of contact for ACP discussions to assist in patient engagement. Nurse navigators already function well in engaging patients with care coordination, patient education and connections to community-based resources. The proposed project is a natural extension of their role and empowers the nurses to use their skills in a new capacity. Studies have shown that the use of nurse navigators in ACP is feasible. ${ }^{35-37}$ Third, we will use the Medicare annual wellness visit to optimise ACP discussions between the patient and their care provider. Our overall hypothesis is that in a primary care setting, a nurse navigator-led ACP pathway will improve ACP documentation within the EHR as compared with usual care and will improve provider-patient communication about goals of care.

\section{MATERIALS AND ANALYSIS Study overview}

This study is a randomised, pragmatic, effectiveness trial for determining better ways to engage vulnerable older adults and their family members in ACP through a nurse navigator-led pathway (intervention arm) versus usual care (control arm; figure 1). A new ACP documentation system that allows for the use of discrete data elements was created into the EHR (Epic Systems Corporation) to allow for easy documentation and tracking of ACP discussions in an outpatient setting. A linkage to the advance directive tab within the EHR was also created along with a new visit type called $\mathrm{ACP},{ }^{38}$ so that all documented goals of care discussions could be found easily in a central location within the EHR. Since nurse navigators were not involved in discussing ACP with patients prior to this study, they were trained in ACP by taking the First Steps ACP Facilitator Certification Course training provided by Respecting Choices (RC) to help facilitate discussions. ${ }^{40}{ }^{41} \mathrm{RC}$ is an internationally recognised, evidence-based model of ACP that creates a healthcare culture of person-centred care: care that honours an individual's goals and values for current and future healthcare. Training consisted of one full day ( 8 hours) working with a trained facilitator to hone interviewing skills related to ACP, and included small group work, didactics, videos, case scenarios, roleplaying, debriefing and self-reflection. A pretest and posttest were also given. In addition, nurse navigators and providers completed a 1 hour training session to review the new ACP documentation programme and observe a short role-play of a goals of care discussion. An automated EHR screening system using existing data within the EHR was created to prescreen eligible patients.

Eligible patients $(n=765)$ were randomised using a 1:1 ratio to either the nurse navigator-led ACP pathway (intervention arm) or usual care (control arm). Permission from primary care providers (PCPs) was obtained to allow the study team to inform their patients about the study using an opt-out strategy. Only those who were randomised to the nurse navigator-led pathway (intervention arm) will be approached for recruitment. Patients who agree to participate will be consented over the telephone and will be screened for eligibility. Nurse navigators will complete 
a brief previsit ACP discussion with the patient over the telephone to help prime and better engage patients in ACP prior to their provider visit. The new ACP telephone documentation system will be used to document these discussions, which will automatically generate a note that will be forwarded to the PCP. After completion of the telephone ACP discussion, patients will be mailed an ACP packet (which will include additional information about ACP and a copy of an advance directive to review) and scheduled for an in-person dyad visit (patient and their surrogate decision-maker or loved one) with their PCP. All visits will be scheduled in conjugation to the patient's Medicare annual wellness visit, unless unable to occur (since can only occur once per year), and if so, will be scheduled as a separate ACP visit alone. PCPs will then complete an ACP dyad visit and document that discussion using the new ACP documentation system. After the visit, patients will be asked to complete a patient engagement survey. ${ }^{42}$ In order to ensure transparency, ACP notes have been systematically programmed to be available to provider's inline with the code status documentation and in the advance directive tab within the EHR.

\section{Study setting}

The geographic region for our intervention is the Piedmont Triad area of North Carolina, which is the northcentral part of the state and contains 12 counties. ${ }^{43}$ The population is estimated at 1.69 million, making it the 30th largest metropolitan area in the USA. In the region, $22.2 \%$ of the residents are African-American, and $15.9 \%$ of the residents are aged 65 and older. ${ }^{43}$ Wake Forest Baptist Health (WFBH) is the only academic medical centre in this 12-county region. WFBH, having recently acquired Cornerstone Health Care, supports more than 200 clinical practice sites in 80 locations throughout central North Carolina. Since 2012, all WFBH locations use an epic-based EHR, which is a single instance, enterprise-wide platform that supports integrated clinical, billing and ancillary applications. Recruitment for this trial occurred at eight separate primary care clinics associated with the WFBH network. Sites were selected in both urban and rural settings across five different counties in North Carolina to help with recruitment of racially and ethnically diverse and low-income populations.

\section{Randomisation procedures}

Patients were randomised $(n=765)$ using a 1:1 ratio to either the nurse navigator-led ACP pathway (intervention arm) or usual care (control arm). We used a Zelen's design ${ }^{44-48}$ for this study, which is a pragmatic clinical trial design whereby all participants are randomised prior to informed consent, and then only patients randomised to the interventional arm will be approached for consent and subsequently enrolled in the intervention group. Note that patients who do not consent to the intervention will still be counted as part of the intervention group under an intent-to-treat paradigm, which necessitates passive ascertainment mechanisms for outcomes (ie, administrative claims or the EHR). One appealing aspect of Zelen's design is that it facilitates estimating real-world effectiveness, as we will be able to estimate the rate at which patients decline to consent for the study, or refuse the nurse navigator intervention, which then factors into overall estimates of effectiveness. In addition, others have pointed out that the Zelen's design is ethical and particularly useful within the context of trials of screening interventions, where the desire is to estimate an effect on the entire population of eligible patients. ${ }^{456}$

\section{Eligibility criteria}

Patients were eligible for this study if they were affiliated with an Accountable Care Organization, were aged 65 and older, had seen their PCP within the past 12 months, who had multimorbidity defined by Charlson Comorbidity Index of 3 or higher, ${ }^{49}$ plus impairments in either physical function (eg, mobility limitation or disability), cognition and/or frailty ${ }^{50}$ (table 1). Their PCP gave permission to study staff to contact patients about the study. Patients were excluded if they had moderate-tosevere hearing loss (due to use of a phone intervention), were non-English speakers (since not all navigators speak a second language, subtleties may have not been conveyed effectively), if no phone number was available, and if they had significant memory impairments based on a Short Portable Mental Status Questionnaire (SPMSQ) score of $\geq 5$ or a score of $\geq 6$ or higher for those with only a grade school education..$^{52}$ Since ACP is an iterative process, participants with prior ACP experiences (eg, an advance directive found with the EHR) were excluded. Patients on hospice, in a long-term care facility, or who transferred care to a different PCP were also excluded from the study.

\section{Recruitment and retention}

We obtained a Health Insurance Portability and Accountability Act waiver to access patients' names, age, race/ ethnicity, gender, primary language, phone numbers, addresses, medical record numbers, diagnoses, lab results, medication lists, payer source, as well as dates of outpatient primary care clinic appointments in the past 2 years, other appointments, hospitalisations and emergency room visits in the past 2 years and the name of patients' outpatient primary care providers. From this data, an automated EHR screening system was created to prescreen eligible patients. This system then generated a list of patients who met our inclusion criteria. Prescreened eligible patients $(\mathrm{n}=765)$ from our eight sites were then randomised using a 1:1 ratio to either the nurse navigator-led ACP pathway (intervention arm) or usual care (control arm).

Nurse navigators will be used to recruit eligible patients for the intervention arm. The nurse navigators were trained in RC, an internationally recognised, evidencebased model of ACP that creates a healthcare culture of person-centred care; care that honours an individual's goals and values for current and future healthcare. ${ }^{4041}$ In addition, nurse navigators received training 
Table 1 Inclusion and exclusion criteria of study participants

\section{Patients}

Inclusion 1. Age 65 or older patients within the WFBH ACO.

criteria 2. Have seen their PCP within the WFBH network in the past 12 months.

3. $\mathrm{CCl}$ of 3 or higher.

4. Impairments in either physical function, cognition and/or frailty defined by:

a. Impairments in physical function:

i. ICD-10 codes for:

1. Falls: V00.141A, V00.312A, W01.110A, W01.198A, W03.XXXA, W05.0XXA, W05.1XXA, W05.2XXA, W06.XXXA, W07.XXXA, W08.XXXA, W10.1XXA, W10.8XXA, W17.81XA, W17.89XA, W18.11XA, W18.30XA, W19.XXXA, R29.6,z91.81

2. Muscular deconditioning: R29.898

3. Physical deconditioning: R53.81

4. Gait abnormality: R26.9, 26.89

5. Impaired physical mobility: $Z 74.09$

6. Difficulty walking: R26.2

7. Debility: R53.81, R54

8. Wheelchair users: Z99.3

ii. Annual wellness visit:

1. Positive falls assessment

2. Impairments in activities of daily living, answer of 'yes' for needing assistance with any of the following:

a. Feeding self, bathing self, dressing self, use of toilet, needing assistive device for walking or cannot walk.

b. Impairments in cognition:

i. $I C D-10$ codes for:

1. Impaired cognition: R41.89

2. Dementia: F01.50, F02.81,F03.90, G30.9, F02.80, G20, G31.83, G31.09, G30.0, G30.1, G30.8, G31.01, G31.09

3. Memory change: R41.3, F06.8

4. $\mathrm{MCl}: \mathrm{G} 31.84$

5. History of memory loss: Z86.59

6. History of short-term memory loss: Z87.898

ii. Annual wellness visit:

1. Answer of 'yes' to either 'has a diagnosis of dementia or cognitive impairment?' and/or 'are there any memory concerns by the patient, others or providers?'

c. Frailty:

i. eFI score $>0.21 .{ }^{50} 5764$

5. English speaking.

6. No documented advance directive in the EHR.

Exclusion 1. Moderate-to-severe hearing loss (due to phone interventions).

criteria 2. Non-English-speaking (not all navigators speak a second language; subtleties may not be conveyed effectively).

3. No phone number available for patient.

4. Moderate/severe cognitive impairment assessed by validated SPMSQ ${ }^{51} 52$

5. Enrolled on hospice, in a long-term care facility or who transferred care to a different PCP.

$\mathrm{ACO}$, Accountable Care Organization; $\mathrm{CCl}$, Charlson Comorbidity Index; eFI, Electronic Frailty Index; EHR, electronic health record; MCI, mild cognitive impairment; PCP, primary care provider; SPMSQ, Short Portable Mental Status Questionnaire; WFBH, Wake Forest Baptist Health.

in the Collaborative Institutional Training Initiative and the protocol. They were added to the research team to recruit, consent patients and complete an initial ACP discussion over the phone. The nurse navigators also will perform a SPMSQ ${ }^{51}$ for patients who are flagged as having an impairment in cognition to rule out patients with moderate to severe dementia.

The nurse navigators will call up to three times to try and recruit a participant. Once a patient consents to participate, the nurse navigator will complete a telephone
ACP visit and then schedule them to see their primary care provider for a dyad ACP in-person visit. Patients will receive a reminder call 1 week prior to their visit. Patients who are are no-shows or cancel their appointment will be called up to three times to try and reschedule their appointment. A missed appointment postcard will be sent as a fourth attempt, and patients will be considered lost to follow-up if after four attempts they cannot be reached. The study team will also be sending Thank You and Appointment Reminder postcards to all participants 
enrolled in the intervention arm. Participants who complete the ACP telephone discussion, the ACP dyad in-person visit and the patient engagement survey will be given a US $\$ 25$ gift card as a token of appreciation for their participation.

\section{Consent procedures}

Our consent was designed to meet the understanding capabilities of our elderly population with a sixth-grade reading level (online supplementary 1 ). The patient and family advisory team reviewed our informed consent and revisions were made as needed. We received approval by our Institutional Review Board (IRB) to obtain verbal consent by phone for patients and a copy of this consent will be mailed to all enrolled participants in the intervention arm. In our informed consent, we stated that the purpose of the study was to find better ways to engage patients in discussing their goals and values with their primary care provider (PCP through ACP. We stated that the study would consist of three steps: (1) to review a few questions about ACP with the nurse navigator over the phone; (2) to meet with their PCP and their caregiver to further discuss ACP; and (3) to complete a patient engagement survey to provide feedback about their ACP conversation with their PCP.

\section{Patient and public involvement}

Our engagement plan calls for meaningful patient, family and stakeholder involvement at every step of the research project-including analysis and dissemination (table 2). The research team includes three sets of stakeholders: (1) The Patient and Family Advisory Panel, which consist of 10 patients or family members/caregivers; (2) The Research Support Team, which consist of four nurse navigators and eight site champions (MD, PA or NP), one from each of the eight community-based clinics participating in the study and (3) The Investigator Team, made up of primary investigators, mentors, analysts and research assistants. All dissemination activities will be led by a group that includes at least one member of each group. This process will ensure that all three sets of stakeholders can share learnings and successes from their own perspective, and that all three groups have buy-in and recognition for their role in the project. Our engagement plan is founded on the principle of meaningful participation. ${ }^{53} 54$ Engaging with key stakeholders can strengthen the understanding of real-world concerns, identify knowledge gaps and barriers and improve knowledge of health inequities in a given community. Teams will meet three times per year and more if needed. Members of the Patient and Family Advisory Panel and Research Support Team will be

Table 2 Engagement plan

\section{Stage}

Barrier assessment for ACP

Research design

Survey design

Conducting the study

Data analysis and interpretation

Dissemination
Patients and family members

\section{Research support team and investigator team}

Patients and family members helped to identify Teams helped to identify and prioritise and prioritise the key barriers to effective ACP. the key barriers to effective ACP from a provider level.

Draft design was presented. Patients and family members had an opportunity to give feedback and reshape study design. They were involved in revising study materials and protocol to ensure feasibility for clinicians and patients.

The investigator team presented our draft patient engagement survey. The patients and family members had final say in survey design.

Patients/families will be involved in recruitment and implementation phase to increase sustained recruitment and ensure study viability.

Patients/families will be presented with preliminary analytic results. They will have the opportunity to suggest new analytic perspectives and to help translate results.

Patients/families identify opportunities to present and shape information about the study, to move away from traditional models of dissemination and to think more creatively about how to get information into the hands of and different audiences.
those who need it. 
compensated equally (annual honoraria of US\$100). Compensation demonstrates recognition of the value of everyone's time, and contributes to the attitude that all members of the research team are valued as contributors to the research project.

\section{Measures and data collection}

\section{Primary and secondary outcomes}

Our primary outcomes are documentation of ACP discussions within the EHR and the quality of ACP discussions. For the purpose of this study, documentation of ACP discussions includes both nurse navigators and PCP's ACP discussion documentation within the EHR. We will measure quality of ACP discussions from two different mechanisms. First, we will use the quality about end-of-life communication (QOC) $)^{55}$ to assess quality of ACP discussion from the patient's perspective through a patient engagement survey. QOC is a 13-item instrument with an overall score and two subscale scores for 'general communication skills' and 'communication about end-of-life care ${ }^{55}$ Scores range from 0 ('poor') to 10 ('absolutely perfect'). Higher scores determine better outcomes. Second, a scoring mechanism was created to measure quality of ACP discussions for both the telephone ACP discussions with the nurse navigator along with primary care provider's ACP visit discussion. Each question listed in the new ACP documentation programme was given a numerical score if the question was answered appropriately. Answers to these questions will be reviewed manually and scored. Telephone ACP discussions has scores ranging from 0 to 8 and provider ACP discussions has scores ranging from 0 to 15 , with higher scores indicating better quality of discussion.

Secondary outcomes were chosen to measure the full process of ACP. We will measure ACP billing code usage (99497, 99498) to help assess ACP discussion rates. We will measure documentation of designated surrogate decision-makers along with advance directive completion rates as another marker to assess ACP documentation rates within the EHR.

Our exploratory outcomes were chosen to measure additional ACP processes along with the impact of ACP. We will be measuring medical scope of treatment completion rates. Patient healthcare utilisation rates will be measured by the number of the following events: inpatient hospitalisations, emergency department (ED) visits, intensive care unit admissions and length of stay, mechanical intubations rates and in-hospital Cardiopulmonary resuscitation (CPR) rates measured in the EHR, along with quality of end-of-life care, which will be measured by after-death bereaved family member interviews. ${ }^{56}$ The interview provides an assessment of patient-focused, family-centred care and assesses overall quality of care received.

\section{Analytic plan}

The primary statistical aim is the comparison of rates at which ACP discussions are documented with the EHR between the nurse navigator and usual care groups. We will use regression techniques for censored time-to-event outcomes to compare the time to documentation of an ACP discussion, including a frailty term (ie, random effect, different from the clinical concept of frailty) to account for correlations between patients with the same primary care physician. ${ }^{57}$ The advantage of a time-toevent analytic framework, versus treating documentation of an ACP discussion as a binary outcome, is that it can account for variable lengths of follow-up and account for the competing risk of death using extensions such as the popular proportional model of Fine and Gray. ${ }^{58}$ Follow-up time for patients without documentation of an ACP discussion will be defined either as of the date of the last in-person encounter within the health system (outpatient, inpatient or ED visit) or as the date of death. Analyses of secondary endpoints (completion of advanced directives, completion of Medical Orders of Scope Treatment forms, utilisation of ACP billing codes, and healthcare utilisation) will similarly use a time-to-event analytic framework. One additional statistical nuance, primarily with healthcare utilisation, is the potential for recurrent events, that is, a patient with multiple ED visits. We will use extensions for time-to-event analyses that can accommodate recurrent events, such as the Mean Cumulative Count estimator ${ }^{59}$ and the regression approach of Prentice et $_{a l}{ }^{60}$

\section{Power and sample size considerations}

Our power estimates are based on standard calculations for time-to-event analyses. ${ }^{61}$ The primary nuance for estimating statistical power is the use of Zelen's prerandomisation design, whereby only patients randomised to the nurse navigator group will be approached for consent. This naturally attenuates any presumed effect of the intervention, as a proportion of patients will not receive the intervention. ${ }^{62}$ Based on a previous randomised trial of ACP strategies conducted within the Veterans Affairs system, we assumed that $44 \%$ of patients randomised to the nurse navigator group will consent to participate. ${ }^{63}$ Furthermore, we assumed that incidence of documented ACP discussions would be $25 \%$ for patients who do not consent or those randomised to usual care. Finally, we assumed a follow-up period of 1 year, that $10 \%$ of patients would be lost to follow-up, and an alpha-level of 0.05 . Based on these assumptions, our initial calculations indicated that a total sample size of 300 patients (150 per group) would provide $>80 \%$ power. However, we subsequently realised a deficiency in these assumptions. Since patients will be randomised prior to consent to the intervention arm, there can be a time lag of up to $\sim 3$ months in between randomisation and initial phone contact for consent. Patients could therefore become ineligible in the interim, for example, by having transitioned to a nursing home or by passing away. We therefore revised our power calculations including an expectation that $20 \%$ of patients in the nurse navigator group would be found ineligible by the time they are contacted, and that 
the incidence of documented ACP discussions within this group would be at most $10 \%$. With an increased sample size of 765 , we expect that $n=135$ of those randomised to the intervention arm will consent to participate. We will have $>80 \%$ power provided that the rate of documented ACP discussions is at least $70 \%$ for participants who consent to the nurse navigator intervention (which implies an overall rate of ACP discussions of $38 \%$ in the nurse navigator arm). If the rate of documented ACP discussions is $30 \%$ in patients who do not consent or are randomised to usual care, then at least $80 \%$ of participants who consent to the nurse navigator intervention will need to have an ACP discussion documented to have $>80 \%$ power (implies an overall rate of ACP discussions of $\sim 44 \%$ in patients randomised to the nurse navigator group).

\section{Ethics and dissemination}

For academic audiences, we will present our findings at scientific meetings and in peer-reviewed research journals. We will also present these results to our patient and family advisory panel. If this study is successful, we will work towards refining and disseminating our study to primary care clinics through the Wake Forest Network and other healthcare systems.

\section{Author affiliations}

${ }^{1}$ Department of Internal Medicine, Section of Gerontology \& Geriatric Medicine, Wake Forest University School of Medicine, Winston-Salem, North Carolina, USA ${ }^{2}$ Department of Biostatistics and Data Science, Division of Public Health Sciences, Wake Forest University School of Medicine, Winston-Salem, North Carolina, USA ${ }^{3}$ Department of Internal Medicine, Wake Forest University School of Medicine, Winston-Salem, North Carolina, USA

${ }^{4}$ Department of Implementation Science, Division of Public Health Sciences, Wake Forest University School of Medicine, Winston-Salem, North Carolina, USA ${ }^{5}$ Department of Internal Medicine,Section of Gerontology \& Geriatric Medicine, Wake Forest Baptist Medical Center, Winston-Salem, North Carolina, USA

\section{Twitter NM Pajewski @NMPajewski}

Acknowledgements The authors would like to acknowledge the editing assistance provided by Indra Newman, PhD from the Wake Forest Clinical and Translational Science Institute (WF CTSI), which is supported by the National Center for Advancing Translational Sciences (NCATS), National Institutes of Health, through Grant Award Number UL1TR001420. The authors would also like to acknowledge our Patient and Family Advisory Panel and our Research Support Team for their assistance with study design and implementation.

Contributors JG, NMP, KEC and JW conceptualised this study. AD and AM contributed in the clinical informatics component of this study. JG and NMP drafted the manuscript. KEC, AD, KF, KGF, AM, CG and JW contributed in editing of the manuscript. All authors approved the final manuscript.

Funding This work was supported by Duke Endowment Health Care Grant and Wake Forest Center for Healthcare Innovation.

Competing interests None declared.

Patient consent for publication Not required.

Ethics approval This study was guided by a patient and family advisory committee comprising of patients, patient advocates and surrogates; site champions consisting of primary care clinic providers, an internal research team, external advisory members, along with the Wake Forest Institutional Review Board. Participant confidentiality will be ensured and anonymity guaranteed.

Provenance and peer review Not commissioned; externally peer reviewed.

Open access This is an open access article distributed in accordance with the Creative Commons Attribution Non Commercial (CC BY-NC 4.0) license, which permits others to distribute, remix, adapt, build upon this work non-commercially, and license their derivative works on different terms, provided the original work is properly cited, appropriate credit is given, any changes made indicated, and the use is non-commercial. See: http://creativecommons.org/licenses/by-nc/4.0/.

ORCID iD

Jennifer Gabbard http://orcid.org/0000-0001-8666-4833

\section{REFERENCES}

1 U.S. Census Bureau. International database. table 094. Midyear population, by age and sex. Available: http://www.census.gov/ population/www/projections/natdet-D1A.htmIExternal Web Site Icon

2 Gómez-Batiste X, Martínez-Muñoz M, Blay C, et al. Prevalence and characteristics of patients with advanced chronic conditions in need of palliative care in the general population: a cross-sectional study. Palliat Med 2014;28:302-11.

3 "Methods for Analysis of the Financing and Use of Long-Term Services and Supports", 2013. Available: https://www.cbo.gov/ publication/44370

4 Schoenborn NL, Cayea D, McNabney M, et al. Prognosis communication with older patients with multimorbidity: assessment after an educational intervention. Gerontol Geriatr Educ 2017;38.

5 Morrison RS. Research priorities in geriatric palliative care: an introduction to a new series. J Palliat Med 2013;16:726-9.

6 Signorielli N. Physical disabilities, impairment and safety,mental illness, and death. In: Mass media images and impact on health: a Sourcebook. Westport, CT: Greenwood Press, 1993: 37-42.

7 Fortin M, Bravo G, Hudon C, et al. Prevalence of multimorbidity among adults seen in family practice. Ann Fam Med 2005;3:223-8.

8 Fortin M, Soubhi H, Hudon C, et al. Multimorbidity's many challenges. BMJ 2007;334:1016-7.

9 Marengoni A, Angleman S, Melis R, et al. Aging with multimorbidity: a systematic review of the literature. Ageing Res Rev 2011;10:430-9.

10 Smith SM, O'Dowd T. Chronic diseases: what happens when they come in multiples? Br J Gen Pract 2007;57:268-70.

11 Taylor AW, Price K, Gill TK, et al. Multimorbidity - not just an older person's issue. Results from an Australian biomedical study. BMC Public Health 2010;10:718.

12 Vogeli C, Shields AE, Lee TA, et al. Multiple chronic conditions: prevalence, health consequences, and implications for quality, care management, and costs. J Gen Intern Med 2007;22 Suppl 3:391-5.

13 Menotti A, Mulder I, Nissinen A, et al. Prevalence of morbidity and multimorbidity in elderly male populations and their impact on 10year all-cause mortality: the fine study (Finland, Italy, Netherlands, elderly). J Clin Epidemiol 2001;54:680-6.

14 Fortin M, Lapointe L, Hudon C, et al. Multimorbidity and quality of life in primary care: a systematic review. Health Qual Life Outcomes 2004;2:51.

15 Townsend A, Hunt K, Wyke S. Managing multiple morbidity in midlife: a qualitative study of attitudes to drug use. BMJ 2003;327.

16 Barnett K, Mercer SW, Norbury M, et al. Epidemiology of multimorbidity and implications for health care, research, and medical education: a cross-sectional study. The Lancet 2012;380:37-43.

17 Smith SM, Soubhi H, Fortin M, et al. Managing patients with multimorbidity: systematic review of interventions in primary care and community settings. BMJ 2012;345:e5205.

18 Ryan A, Wallace E, O'Hara P, et al. Multimorbidity and functional decline in community-dwelling adults: a systematic review. Health Qual Life Outcomes 2015;13:168.

19 lezzoni LI. Multiple chronic conditions and disabilities: implications for health services research and data demands. Health Serv Res 2010;45:1523-40.

20 Quiñones AR, Markwardt S, Botoseneanu A. Multimorbidity combinations and disability in older adults. GERONA 2016;71:823-30.

21 Kogan AC, Wilber K, Mosqueda L. Person-Centered care for older adults with chronic conditions and functional impairment: a systematic literature review. J Am Geriatr Soc 2016;64:e1-7.

22 Connors MH, Sachdev PS, Kochan NA, et al. Cognition and mortality in older people: the Sydney memory and ageing study. Age Ageing 2015;44:1049-54.

23 Bunn F, Goodman C, Burn A-M. Multimorbidity and frailty in people with dementia. Nursing Standard 2015;30:45-50.

24 Bunn F, Burn A-M, Goodman C, et al. Comorbidity and dementia: a scoping review of the literature. BMC Med 2014;12:192.

25 Martín Lesende I, Mendibil Crespo LI, Castaño Manzanares S, et al. Functional decline and associated factors in patients with 
multimorbidity at 8 months of follow-up in primary care: the functionality in pluripathological patients (FUNCIPLUR) longitudinal descriptive study. BMJ Open 2018;8:e022377.

26 Campbell SE, Seymour DG, Primrose WR, et al. A systematic literature review of factors affecting outcome in older medical patients admitted to hospital. Age Ageing 2004;33:110-5.

27 Hagerty RG, Butow PN, Ellis PM, et al. Communicating with realism and hope: incurable cancer patients' views on the disclosure of prognosis. JCO 2005;23:1278-88.

28 Guiding principles for the care of older adults with multimorbidity: an approach for clinicians: American geriatrics Society expert panel on the care of older adults with multimorbidity. J Am Geriatr Soc 2012;60:E1-25.

29 Herrin J, Harris KG, Kenward K, et al. Patient and family engagement: a survey of US Hospital practices. BMJ Qual Saf 2016;25:182-9.

30 Asakawa T, Koyano W, Ando T, et al. Effects of functional decline on quality of life among the Japanese elderly. Int J Aging Hum Dev 2000;50:319-28.

31 Stineman MG, Xie D, Pan Q, et al. All-Cause 1-, 5-, and 10-year mortality in elderly people according to activities of daily living stage. $J$ Am Geriatr Soc 2012;60:485-92.

32 KritchevskY SB, Williamson J. Putting function first. J Nutr Health Aging 2014:18:467-8.

33 Mc Cord KA, Ewald H, Ladanie A, et al. Current use and costs of electronic health records for clinical trial research: a descriptive study. CMAJ Open 2019;7:E23-32.

34 Li G, Sajobi TT, Menon BK, et al. Registry-Based randomized controlled trials- what are the advantages, challenges, and areas for future research? J Clin Epidemiol 2016;80:16-24.

35 Rocque GB, Dionne-Odom JN, Sylvia Huang C-H, et al. Implementation and impact of patient lay Navigator-Led advance care planning conversations. J Pain Symptom Manage 2017;53:682-92.

36 Niranjan SJ, Huang C-HS, Dionne-Odom JN, et al. Lay Patient Navigators' Perspectives of Barriers, Facilitators and Training Needs in Initiating Advance Care Planning Conversations With Older Patients With Cancer. J Palliat Care 2018;33:70-8.

37 Rocque GB, Partridge EE, Pisu M, et al. The patient care connect program: transforming health care through lay navigation. JOP 2016;12:e633-42.

38 Turley M, Wang S, Meng D. Impact of a care directives activity TAB in the electronic health record on documentation of advance care planning. Perm J 2016;20:43-8.

39 Huber MT, Highland JD, Krishnamoorthi VR, et al. Utilizing the electronic health record to improve advance care planning: a systematic review. Am J Hosp Palliat Care 2018;35:532-41.

40 Moorman SM, Carr D, Kirchhoff KT, et al. An assessment of social diffusion in the respecting choices advance care planning program. Death Stud 2012;36:301-22.

41 Rietjens JAC, Korfage IJ, Dunleavy L, et al. Advance care planning a multi-centre cluster randomised clinical trial: the research protocol of the action study. BMC Cancer 2016;16:264.

42 Sudore RL, Heyland DK, Barnes DE, et al. Measuring advance care planning: optimizing the advance care planning engagement survey. J Pain Symptom Manage 2017;53:669-81.

43 United States census Burea, North Carolina. Available: https://www. census.gov/quickfacts/fact/table/NC/PST045218

44 Zelen M. A new design for randomized clinical trials. N Engl J Med 1979;300:1242-5.
45 Torgerson DJ, Roland M. What is Zelen's design? BMJ 1998;316.

46 Torgerson D. The use of Zelen's design in randomised trials. BJOG 2004;111:2

47 House A, Knapp P. Informed consent. trials that use Zelen's procedure should be acceptable. BMJ 1997;315.

48 Adamson J, Cockayne S, Puffer S, et al. Review of randomised trials using the post-randomised consent (Zelen's) design. Contemp Clin Trials 2006;27:305-19.

49 Charlson ME, Pompei P, Ales KL, et al. A new method of classifying prognostic comorbidity in longitudinal studies: development and validation. J Chronic Dis 1987;40:373-83.

50 Clegg A, Bates C, Young J, et al. Development and validation of an electronic frailty index using routine primary care electronic health record data. Age Ageing 2016;45:353-60.

51 Erkinjuntti T, Sulkava R, Wikström J, et al. Short portable mental status questionnaire as a screening test for dementia and delirium among the elderly. J Am Geriatr Soc 1987;35:412-6.

52 Castanho TC, Amorim L, Zihl J, et al. Telephone-based screening tools for mild cognitive impairment and dementia in aging studies: a review of validated instruments. Front Aging Neurosci 2014;6:16.

53 Higgins T, Larson E, Schnall R. Unraveling the meaning of patient engagement: a concept analysis. Patient Educ Couns 2017:100:30-6.

54 de Wit M, Cooper C, Tugwell P, et al. Practical guidance for engaging patients in health research, treatment guidelines and regulatory processes: results of an expert group meeting organized by the WORLD HEALTH ORGANIZATION (WHO) and the European Society for clinical and economic aspects of osteoporosis, osteoarthritis and musculoskeletal diseases (ESCEO). Aging Clin Exp Res 2019;31:905-15.

55 Engelberg R, Downey L, Curtis JR. Psychometric characteristics of a quality of communication questionnaire assessing communication about end-of-life care. J Palliat Med 2006;9:1086-98.

56 Teno JM, Clarridge B, Casey V, et al. Validation of toolkit AfterDeath bereaved family member interview. J Pain Symptom Manage 2001;22:752-8.

57 Glidden DV, Vittinghoff E. Modelling clustered survival data from multicentre clinical trials. Stat Med 2004;23:369-88.

58 Fine JP, Gray RJ. A proportional hazards model for the Subdistribution of a competing risk. J Am Stat Assoc 1999;94:496-509. \&.

59 Dong $\mathrm{H}$, Robison LL, Leisenring WM, et al. Estimating the burden of recurrent events in the presence of competing risks: the method of mean cumulative count. Am J Epidemiol 2015;181:532-40.

60 Prentice RL, Williams BJ, Peterson AV. On the regression analysis of multivariate failure time data. Biometrika 1981;68:373-9.

61 Julious SA. Sample sizes for clinical trials. Chapman \& Hall/CRC, Boca Raton, FL; 2010.

62 Reeves D, Howells K, Sidaway M, et al. The cohort multiple randomized controlled trial design was found to be highly susceptible to low statistical power and internal validity biases. J Clin Epidemiol 2018;95:111-9.

63 Sudore RL, Boscardin J, Feuz MA, et al. Effect of the prepare website vs an Easy-to-Read advance directive on advance care planning documentation and engagement among veterans: a randomized clinical trial. JAMA Intern Med 2017;177:1102-9.

64 Pajewski NM, Lenoir K, Wells BJ, et al. Frailty screening using the electronic health record within a Medicare accountable care organization. J Gerontol A Biol Sci Med Sci 2019;74:1771-7. 